\title{
Temperature tolerance and biogeography of seaweeds: The marine algal flora of Helgoland (North Sea) as an example*
}

\author{
K. Lüning
}

Biologische Anstalt Helgoland (Zentrale); Notkestraße 31, D-2000 Hamburg 52, Federal
Republic of Germany

ABSTRACT: Temperature tolerance (1 week exposure time) was determined at intervals during two successive years in 54 dominant marine benthic algae growing near Helgoland (North Sea). Seawater temperatures near Helgoland seasonally range between $3^{\circ} \mathrm{C}$ (in some years $0^{\circ}$ ) and $18^{\circ} \mathrm{C}$. All algae survived $0^{\circ} \mathrm{C}$, and none $33^{\circ} \mathrm{C}$. Among the brown algae, Chorda tomentosa was the most sensitive species surviving only $18^{\circ} \mathrm{C}$, followed by the Laminaria spp. surviving $20^{\circ}$, however not $23^{\circ} \mathrm{C}$. Fucus spp. and Cladostephus spongiosus were the most heat-tolerant brown algae, surviving $28^{\circ} \mathrm{C}$. Among the red algae, species of the Delesseriaceae (Phycodrys rubens, Membranoptera alata) ranged on the lower end with a maximum survival temperature of $20^{\circ} \mathrm{C}$, whereas the representatives of the Phyllophoraceae (Ahnfeltia plicata, Phyllophora truncata, P. pseudoceranoides) exhibited the maximum heat tolerance of the Helgoland marine algal flora with survival at $30^{\circ} \mathrm{C}$. The latter value was also achieved by Codium fragile, Bryopsis hypnoides and Enteromorpha prolifera among the green algae, whereas the Acrosiphonia spp. survived only $20^{\circ} \mathrm{C}$, and Monostroma undulatum only $10^{\circ} \mathrm{C}$, not $15^{\circ} \mathrm{C}$. Seasonal shifts of heat tolerance of up to $5^{\circ} \mathrm{C}$ were detected, especially in Laminaria spp. and Desmarestia aculeata. The majority of the dominant marine algal species of the Helgoland flora occurs in the Arctic, and it is hypothesized that also there the upper lethal limits of these species may hardly have changed even today. The data presented should provide a base for further analysis of the causes of geographical distribution of the North Atlantic algal species, but have still to be supplemented with similar investigations on other coasts, and supplemented with determinations of temperature requirements throughout the life cycle.

\section{INTRODUCTION}

Investigations on the temperature tolerance of the components of local algal floras at as many stations as possible may increase our knowledge of at least three different aspects of the geographical distribution of these components.

(1) On the basis of such studies one may recognize the species whose upper lethal limits are so near to the locally, yearly occurring temperature maxima that these species at the given locality just survive. On the other hand, one may find those species whose upper lethal limits may lie up to $10^{\circ} \mathrm{C}$ above the actually occurring summer temperatures. In the latter case lower temperature demands for growth or reproduction may

\footnotetext{
- Paper presented at the Seaweed Biogeography Workshop of the International Working Group on Seaweed Biogeography, held from 3-7 April 1984 at the Department of Marine Biology, University of Groningen (The Netherlands). Convenor: C. van den Hoek
} 
reduce the distribution area in comparison to an area constructed on the base of tolerance limits. In addition, the case of a species with a very wide temperature survival range may also mean that this species has not genetically adapted to the local temperature conditions. Information on temperature tolerance and on the temperature demands of reproduction and growth may be used for comparisons of the species' actual distribution areas with ocean isotherms, as has been demonstrated by van den Hoek (1982a, $1982 \mathrm{~b}$ ) and in several papers of the present volume (Cambridge et al., 1984; McLachlan \& Bird, 1984; Rietema \& van den Hoek, 1984; Yarish et al., 1984).

(2) If tests on temperature tolerance are performed throughout the year, one may discriminate whether or not, and to what extent, an algal species can shift its survival limits during the year.

(3) Comparisons of the temperature survival ranges of a given species at different localities may reveal whether or not a given species throughout its distribution area is genetically flexible enough to form temperature ecotypes and by this to enlarge its distribution area. A first result in this respect was obtained by Bolton (1983) who found that the upper survival temperatures of Ectocarpus siliculosus in a range of samples from the Arctic to warm temperate regions differed by as much as $10^{\circ} \mathrm{C}$. On the other hand temperature requirements for survival of the gametophytes of several cold temperate Laminaria spp. are quite similar in the northern hemisphere (Bolton \& Lüning, 1983).

(4) Finally, since the fossilization potential of seaweeds is so small, one may find that the temperature characteristics of species, genera or even families have been so stable over millions of years that the temperature behaviour may be used as a "fossil trait". For instance, the Arctic coasts are today inhabited largely by algal species which occur in the North Atlantic, partly also in the North Pacific. In contrast, the peculiar and rich seaweed flora of the North Pacific hardly entered the Arctic through the Bering Strait. The latter was open for most of the last 4 millions years, and only closed at low sea levels during the pleistocene glaciations (Briggs, 1974; Frakes, 1979; van den Hoek, 1975). This means that the seaweeds in the North Atlantic, with its wide open connection to the Arctic Ocean, were exposed for longer periods to lower temperatures than the seaweeds of the North Pacific, when the process of climate deterioration started by the end of the Tertiary and culminated in the Ice Age. Will we still be able to detect today a greater sensitivity in North Pacific algae to temperatures near or below the freezing point of seawater in comparison to North Atlantic algae which had a greater chance to develop cold water forms and possibly, therefore, still today dominate the Arctic?

At present, the few investigations on temperature tolerance, and the still fewer ones on temperature demands for growth and reproduction of the components of local algal floras are hardly enlightening on the four aspects presented above. It is hoped that the results presented in this paper will stimulate more investigations of this kind on other coasts also. The only extensive investigations on temperature tolerance of different seaweed species from various geographical locations are those by Biebl $(1958,1962$, 1968, 1970; Table 1), which give an idea of the different temperature ranges tolerated by seaweeds from Greenland, Brittany, the Mediterranean and from Puerto Rico. There are, however, three major drawbacks to Biebl's work on temperature tolerance. First, he had to select a limited number of species from each location, and seldom does one find data for the same species investigated at different locations. Secondly, survival has been tested after an exposure time of only $12 \mathrm{~h}$ to the different temperatures, and, as will be 
seen in the present paper, this approach gives upper lethal limits that are too high and misleading in comparison to such limits determined on the basis of an exposure time of a week. Thirdly, the decision on the status "dead or alive" requires a safe criterion, such as a measurable photosynthetic rate, whereas Biebl had to rely on quick, but somewhat uncertain methods, such as cytomorphological criteria.

Table 1. Temperature tolerance of benthic marine algae from different geographical regions (data from Biebl 1958, 1962, 1968, 1970)

\begin{tabular}{|llcccc|}
\hline $\begin{array}{c}\text { Type of } \\
\text { biogeographical } \\
\text { region }\end{array}$ & Location & $\begin{array}{c}\text { Annual } \\
\text { span }\end{array}$ & \multicolumn{2}{c|}{$\begin{array}{c}\text { Temperature survival range } \\
\text { after } 12 \mathrm{~h} \text { exposure }\end{array}$} \\
& $\left({ }^{\circ} \mathrm{C}\right)$ & $\begin{array}{c}\text { Eulittoral species } \\
\left({ }^{\circ} \mathrm{C}\right)\end{array}$ & $\begin{array}{c}\text { Sublittoral species } \\
\left({ }^{\circ} \mathrm{C}\right)\end{array}$ \\
\hline Arctic & W-Greenland & 0 to 6 & -10 to 35 & -2 to $22(24)$ \\
Warm temperature & Brittany & 10 to 16 & -8 to $30(35)$ & $-2(0)$ to $27(30)$ \\
Warm temperature & Naples & 14 to 24 & -7 to 35 & $1(2)$ to $27(30)$ \\
Tropical & Puerto Rico & 26 to 28 & -2 to $35(40)$ & $+14(5)$ to $35(32)$ \\
\hline
\end{tabular}

\section{MATERIAL AND METHODS}

Algae were collected at intervals during two successive years in the eulittoral zone or by divers in the sublittoral zone of Helgoland, North Sea. Erlenmeyer flasks (1 1) were filled with seawater and immersed in the water baths of Colora cryostats (Colora Messtechnik, Lorch, FRG). The water baths were maintained at $18^{\circ}, 20^{\circ}, 23^{\circ}, 25^{\circ}, 28^{\circ}$, $30^{\circ}, 33^{\circ}$, and $35^{\circ} \mathrm{C}\left(\right.$ all $\left.\pm 0.1 \mathrm{C}^{\circ}\right)$. Constant temperature rooms (all $\pm 0.5^{\circ} \mathrm{C}$ ) were used for the temperatures of $0^{\circ}, 5^{\circ}, 10^{\circ}, 15^{\circ} \mathrm{C}$. The flasks contained each a quantity of $0.5-5 \mathrm{~g}$ of fresh weight of each species, and exposure time was 1 week. The medium was replaced every 2 days by seawater of the appropriate temperature. Temperature of the water baths was measured daily, and deviations were less than $\pm 0.1^{\circ} \mathrm{C}$. Cool-white fluorescent light (Osram-L $65 \mathrm{~W} / 19$, Daylight 5000 De-Luxe) was used, and the plants received continuously a photon flux density of $30 \mu \mathrm{E} \mathrm{m} \mathrm{m}^{-2} \mathrm{~s}^{-1}$.

Survival after 1 week's exposure at the different temperatures was assessed by measuring photosynthesis by means of an oxygen electrode (Yellow Springs Instrument Co., Ohio). For this purpose the experimental algal material $(0.3-1 \mathrm{~g}$ of fresh weight) was placed into glass-stoppered flasks ( $100 \mathrm{ml}$ of volume) completely filled with seawater of known oxygen content, and exposed for $16 \mathrm{~h}$ to cool-white fluorescent light at a photon flux density of $50 \mu \mathrm{E} \mathrm{m} \mathrm{m}^{-2} \mathrm{~s}^{-1}$, in a constant temperature room at $10^{\circ} \mathrm{C}$. The oxygen content of a 4 -ml-subsample of the seawater in each bottle ( 2 replicates) was then determined in a measuring tube equipped with an oxygen electrode and magnetic bar. If algae were alive, the oxygen content of the seawater would have risen considerably (up to $200 \%$ of oxygen saturation). In the case of dead algae the oxygen content did not increase, but in most cases decreased considerably due to bacterial activity in the water containing the dead algae. The boundary between "alive" and "dead" was always sharp. For example, in an experiment with Ahnfeltia plicata, the oxygen values in the bottles which were exposed to $0-28^{\circ} \mathrm{C}$ were all in the range of $120-160 \%$ of the 
saturation value. The oxygen values of samples which were exposed to 30 and $33^{\circ} \mathrm{C}$ were in the range of $10-15 \%$ of the saturation value.

\section{RESULTS AND DISCUSSION}

The seasonal course of the seawater temperature in the surface waters near Helgoland was almost identical during the two years, in which the experiments were conducted (Table 2). The annual minimum in February was as a monthly mean value around $3^{\circ} \mathrm{C}\left(2.2^{\circ} \mathrm{C}\right.$ as a minimum on a few days), and the annual maximum in August around $16.5^{\circ} \mathrm{C}\left(17.9^{\circ} \mathrm{C}\right.$ as an occasional maximum). The results on temperature tolerance, which are given in Tables 3-5, were therefore arranged in such a way that experiments performed in the same month, but in the two different years, appear one after the other. This arrangement shows that identical results were obtained in both years.

Table 2. Seawater temperatures (surface) near Helgoland (from Biologische Anstalt Helgoland, Jahresbericht 1980, Jahresbericht 1981)

\begin{tabular}{|cccccccccccccc|}
\hline Year & $\mathrm{J}$ & $\mathrm{F}$ & $\mathrm{M}$ & $\mathrm{A}$ & $\mathrm{M}$ & $\mathrm{J}$ & $\mathrm{J}$ & $\mathrm{A}$ & $\mathrm{S}$ & $\mathrm{O}$ & $\mathrm{N}$ & $\mathrm{D}$ \\
& & & & & & & & \\
\hline & & & & & & \\
\hline 1980 & 4.9 & 3.4 & 3.3 & 5.3 & 9.0 & 12.3 & 14.6 & 16.7 & 16.1 & 13.4 & 9.4 & 6.4 \\
1981 & 3.8 & 3.1 & 3.8 & 6.0 & 8.5 & 12.3 & 15.2 & 16.6 & 16.1 & 13.1 & 9.1 & 5.0 \\
\hline
\end{tabular}

All investigated algal species survived 1 week's exposure at $0^{\circ} \mathrm{C}$, which is no surprise, since temperatures around zero occur near Helgoland at intervals of several years, although not during the two years of the investigations. Survival at temperatures between $0^{\circ} \mathrm{C}$ and the freezing point of seawater at $-2{ }^{\circ} \mathrm{C}$ has not been tested, and so it remains an open question, whether or not characteristic differences may still be found for the various species in this temperature range. Hence, in the following only the speciesspecific differences in heat tolerance will be treated.

It should be realized that in the course of the present study all survival temperatures were determined in the submerged condition, and it is well-known for various droughttolerant cryptogams including some intertidal seaweeds that the upper lethal limit increases with decreasing water content (Kappen, 1981). According to Schramm (1968) the heat tolerance of $F$. vesiculosus which has been dehydrated to $30 \%$ of its original water content, rises by $5^{\circ} \mathrm{C}$ in comparison to fully hydrated thalli. Bangia fuscopurpurea from Naples survived $42^{\circ} \mathrm{C}$ for $12 \mathrm{~h}$, if dehydrated, however only $30^{\circ}$, if submerged (Biebl, 1939). One might expect that Blidingia minima, due to its surprisingly low heat tolerance in the submerged state (dead at $28^{\circ} \mathrm{C}$; Table 5), may in fact require emersion and water loss for survival near its southern limit.

It is evident from the present study that in early spring the upper survival temperature of new-grown tissue of Laminaria saccharina and L. digitata was by $2{ }^{\circ} \mathrm{C}$ lower than in summer, and in L. hyperborea, as well as in Desmarestia aculeata this difference between spring and summer material amounted to $5^{\circ} \mathrm{C}$ (Table 3). In the latter two species the heat tolerance of old and new thallus parts differed even in the same month by $2^{\circ} \mathrm{C}$. These facts were already indicated by the early work of Lampe (1935) and 
Table 3. Phaeophyceae. Temperature tolerance of dominant species near Helgoland in two successive years. $\times \times \times=$ plants alive (with measurable photosynthesis)

\begin{tabular}{|c|c|c|c|c|c|c|c|c|c|c|c|c|c|}
\hline \multirow{2}{*}{\multicolumn{3}{|c|}{ Species }} & \multicolumn{11}{|c|}{ Temperature during one week $\left({ }^{\circ} \mathrm{C}\right)$} \\
\hline & & & 0 & 5 & 10 & 15 & 18 & 20 & 23 & 25 & 28 & 30 & 33 \\
\hline \multicolumn{14}{|c|}{ Chorda tomentosa } \\
\hline & May & 80 & $x \times x$ & $\times \times x$ & $\times \times \times$ & $x \times x$ & $x \times x$ & & & & & & \\
\hline & Jun & 81 & $x \times x$ & $\times \times \times$ & $x \times x$ & $x \times x$ & $\times \times \times$ & & & & & & \\
\hline \multicolumn{14}{|c|}{ Chorda filum } \\
\hline & Jun & 80 & $x \times x$ & $\times \times \times$ & $x \times x$ & $\times \times x$ & $x \times x$ & $x \times x$ & & & & & \\
\hline & Jul & 81 & $x \times x$ & $\times \times \times$ & $x \times x$ & $\times \times x$ & $x \times x$ & $x \times x$ & & & & & \\
\hline \multicolumn{14}{|c|}{ Laminaria saccharina } \\
\hline & Jan & 81 & $x \times x$ & $x \times x$ & $x \times x$ & $\times \times \times$ & $\times \times \times$ & $\times \times \times$ & & & & & \\
\hline & Feb & 81 & $x \times x$ & $\times \times \times$ & $\times \times \times$ & $x \times x$ & $x \times x$ & & & & & & \\
\hline & Mar & 81 & $\times \times \times$ & $x \times x$ & $x \times \times$ & $\times \times x$ & $x \times \times$ & & & & & & \\
\hline & Apr & 81 & $x \times x$ & $x \times x$ & $\times \times \times$ & $\times \times x$ & $x \times x$ & & & & & & \\
\hline & May & 80 & $\times \times \times$ & $\times \times \times$ & $x \times \times$ & $x \times x$ & $x \times x$ & $x \times x$ & & & & & \\
\hline & Jul & 80 & $x \times x$ & $x \times x$ & $\times \times \times$ & $x \times x$ & $\times \times \times$ & $\times \times \times$ & & & & & \\
\hline & Oct & 80 & $x \times x$ & $x \times x$ & $x \times x$ & $x \times x$ & $x \times x$ & $x \times x$ & & & & & \\
\hline & Oct & 81 & $x \times x$ & $x \times x$ & $x \times x$ & $x \times x$ & $x \times x$ & $x \times x$ & & & & & \\
\hline & Nov & 80 & $x \times x$ & $x \times x$ & $x \times x$ & $\times \times x$ & $x \times x$ & $x \times x$ & & & & & \\
\hline \multicolumn{14}{|c|}{ Laminaria digitata } \\
\hline & Jan & 81 & $\times \times \times$ & $\times \times \times$ & $\times \times \times$ & $x \times x$ & $\times \times \times$ & & & & & & \\
\hline & Feb & 81 & $x \times x$ & $x \times x$ & $\times \times \times$ & $\times \times \times$ & $x \times x$ & & & & & & \\
\hline & Mar & 81 & $\times \times \times$ & $x \times x$ & $x \times \times$ & $\times \times x$ & $x \times x$ & & & & & & \\
\hline Old & Apr & 81 & $x \times x$ & $\times \times \times$ & $x \times x$ & $x \times x$ & $\times \times \times$ & $x \times x$ & & & & & \\
\hline \multirow{5}{*}{ New } & Apr & 81 & $x \times x$ & $\times \times \times$ & $\times \times \times$ & $x \times x$ & $\times \times \times$ & $x \times x$ & & & & & \\
\hline & Jun & 80 & $x \times x$ & $x \times x$ & $\times \times \times$ & $\times \times \times$ & $\times \times \times$ & $\times \times \times$ & & & & & \\
\hline & Jun & 81 & $\times \times \times$ & $x \times x$ & $\times \times \times$ & $\times \times x$ & $x \times x$ & $x \times x$ & & & & & \\
\hline & Jul & 81 & $x \times x$ & $x \times x$ & $x \times x$ & $\times \times \times$ & $x \times x$ & $x \times x$ & & & & & \\
\hline & Nov & 80 & $\times \times \times$ & $\times \times \times$ & $x \times \times$ & $x \times x$ & $x \times x$ & $x \times \times$ & & & & & \\
\hline \multicolumn{14}{|c|}{ Laminaria hyperborea } \\
\hline Old & Jan & 81 & $\times \times \times$ & $\times \times x$ & $x \times x$ & $x \times x$ & $\times \times x$ & $x \times x$ & & & & & \\
\hline Old & Feb & 81 & $\times \times \times$ & $x \times x$ & $\times \times \times$ & $\times \times \times$ & $\times \times \times$ & & & & & & \\
\hline New & Feb & 81 & $\times \times \times$ & $x \times x$ & $x \times x$ & $\times \times \times$ & & & & & & & \\
\hline Old & Apr & 81 & $x \times x$ & $\times \times x$ & $\times \times \times$ & $x \times x$ & $x \times \times$ & $x \times x$ & & & & & \\
\hline New & Apr & 81 & $\times \times \times$ & $\times \times \times$ & $\times \times \times$ & $\times \times \times$ & $\times \times \times$ & & & & & & \\
\hline Old & Jun & 81 & $x \times \times$ & $x \times x$ & $\times \times \times$ & $x \times x$ & $\times \times \times$ & $\times \times x$ & & & & & \\
\hline \multirow[t]{4}{*}{ New } & Jun & 81 & $\times \times \times$ & $x \times x$ & $\times \times \times$ & $\times \times \times$ & $\times \times \times$ & $\times \times \times$ & & & & & \\
\hline & Jul & 81 & $\times x \times$ & $x \times x$ & $\times \times \times$ & $x \times x$ & $x \times x$ & $\times \times \times$ & & & & & \\
\hline & Oct & 81 & $x \times x$ & $\times \times \times$ & $x \times x$ & $x \times x$ & $\times \times \times$ & $x \times x$ & & & & & \\
\hline & Nov & 80 & $\times \times \times$ & $\times \times \times$ & $\times \times \times$ & $\times \times \times$ & $\times \times \times$ & $\times \times \times$ & & & & & \\
\hline \multicolumn{14}{|c|}{ Desmarestia aculeata } \\
\hline & Feb & 81 & $\times \times \times$ & $\times \times \times$ & $x \times x$ & $x \times x$ & $x \times x$ & $x \times x$ & & & & & \\
\hline Old & Apr & 81 & $x \times x$ & $x \times x$ & $\times \times \times$ & $x \times x$ & $\times \times \times$ & $x \times x$ & & & & & \\
\hline New & Apr & 81 & $x \times x$ & $x \times x$ & $x \times x$ & $x \times x$ & $x \times x$ & & & & & & \\
\hline Old & May & 80 & $x \times x$ & $\times \times \times$ & $\times \times \times$ & $x \times x$ & $\times \times x$ & $x \times x$ & & & & & \\
\hline New & May & 80 & $x \times x$ & $x \times x$ & $\times \times \times$ & $x \times x$ & $\times \times \times$ & & & & & & \\
\hline Old & Jun & 81 & $\times \times \times$ & $\times \times \times$ & $\times \times \times$ & $x \times \times$ & $x \times x$ & $\times \times \times$ & & & & & \\
\hline \multirow[t]{6}{*}{ New } & Jun & 81 & $x \times x$ & $x \times x$ & $x \times \times$ & $\times \times \times$ & $x \times x$ & $x \times x$ & & & & & \\
\hline & Jul & 80 & $x \times x$ & $x \times x$ & $x \times x$ & $x \times x$ & $x \times x$ & $x \times x$ & & & & & \\
\hline & Jul & 81 & $x \times \times$ & $\times \times \times$ & $\times \times \times$ & $\times \times \times$ & $\times \times \times$ & $\times \times \times$ & & & & & \\
\hline & Sep & 81 & $x \times x$ & $x \times x$ & $x \times x$ & $x \times x$ & $\times \times x$ & $\times \times x$ & $x \times \times$ & & & & \\
\hline & Oct & 80 & $x \times x$ & $x \times x$ & $\times \times x$ & $x \times x$ & $x \times x$ & $\times \times \times$ & $x \times x$ & & & & \\
\hline & Nov & 80 & $x \times x$ & $\times \times \times$ & $x \times x$ & $\times \times \times$ & $x \times x$ & $x \times x$ & $x \times x$ & & & & \\
\hline
\end{tabular}


Table 3. Phaeophyceae (continued)

Species

Temperature during one week $\left({ }^{\circ} \mathrm{C}\right)$

\begin{tabular}{lllllllllll}
0 & 5 & 10 & 15 & 18 & 20 & 23 & 25 & 28 & 30 & 33 \\
\hline
\end{tabular}

\section{Desmarestia viridis}

Jun $80 \times \times \times \times \times \times \times \times \times \times \times \times \times \times \times \times \times \times$

\section{Chordaria flagelliformis}

Aug $81 \times \times \times \times \times \times \times \times \times \times \times \times \times \times \times \times \times \times \times \times$

Petalonia fascia

May $81 \times \times \times \times \times \times \times \times \times \times \times \times \times \times \times \times \times \times \times \times$

\section{Scytosiphon lomentaria}

Jun $80 \times \times \times \times \times \times \times \times \times \times \times \times \times \times \times \times \times \times \times \times \times \times$

\section{Pylaiella litoralis}

Aug $81 \quad \times \times \times \times \times \times \times \times \times \times \times \times \times \times \times \times \times \times \times \times \times \times \times \times$

Dec $81 \times \times \times \times \times \times \times \times \times \times \times \times \times \times \times \times \times \times \times \times \times \times \times$

Sphacelaria radicans

Aug $81 \times \times \times \times \times \times \times \times \times \times \times \times \times \times \times \times \times \times \times \times \times \times \times \times$

Dec $81 \times \times \times \times \times \times \times \times \times \times \times \times \times \times \times \quad \times \times \times \quad \times \times \times \quad \times \times \times$

Halidrys siliquosa

Jul $80 \times \times \times \times \times \times \times \times \times \times \times \times \times \times \times \times \times \times \times \times \times \times \times$

Fucus serratus

Jun $81 \times \times \times \times \times \times \times \times \times \times \times \times \times \times \times \times \times \times \times \times \times \times \times$

Jul $80 \quad \times \times \times \times \times \times \times \times \times \times \times \times \times \times \times \times \times \times \times \times \times \times \times \times$

Jul $81 \times \times \times \times \times \times \times \times \times \times \times \times \times \times \times \times \times \times \times \times \times \times \times \times$

Aug $80 \times \times \times \times \times \times \times \times \times \times \times \times \times \times \times \times \times \times \times \times \times \times \times$

Oct $81 \times \times \times \times \times \times \times \times \times \times \times \times \times \times \times \times \times \times \times \times \times \times \times \times$

Ascophyllum nodosum

Feb $81 \times \times \times \times \times \times \times \times \times \times \times \times \times \times \times \times \times \times \times \times \times \times \times \times$

May $80 \times \times \times \times \times \times \times \times \times \times \times \times \times \times \times \times \times \times \times \times \times \times \times$

Jul $81 \times \times \times \times \times \times \times \times \times \times \times \times \times \times \times \times \times \times \times \times \times \times \times \times \times \times \times$

Dec $80 \times \times \times \times \times \times \times \times \times \times \times \times \times \times \times \times \times \times \times \times \times \times \times \times \times \times \times$

\section{Fucus vesiculosus}

Feb $81 \times \times \times \times \times \times \times \times \times \times \times \times \times \times \times \times \times \times \times \times \times \times \times \times \times \times \times$

May $81 \times \times \times \times \times \times \times \times \times \quad \times \times \times \quad \times \times \times \quad \times \times \times \quad \times \times \times \quad \times \times \times \times \times \times$

Aug $80 \times \times \times \times \times \times \quad \times \times \times \quad \times \times \times \quad \times \times \times \times \times \times \times \times \times \times \times \times \times \times \times$

Oct $81 \times \times \times \quad \times \times \times \times \times \times \quad \times \times \times \quad \times \times \times \quad \times \times \times \quad \times \times \times \quad \times \times \times \times \times \times$

Dec $80 \times \times \times \times \times \times \times \times \times \times \times \times \times \times \times \times \times \times \times \times \times \times \times \times \times \times \times$

Fucus spiralis

Feb $81 \times \times \times \times \times \times \times \times \times \times \times \times \times \times \times \times \times \times \times \times \times \times \times \times \times \times \times$

May $81 \times \times \times \quad \times \times \times \quad \times \times \times \quad \times \times \times \quad \times \times \times \quad \times \times \times \quad \times \times \times \quad \times \times \times \times \times \times$

Jul $81 \times \times \times \times \times \times \times \times \times \times \times \times \times \times \times \times \times \times \times \times \times \times \times \times \times$

Oct $81 \times \times \times \quad \times \times \times \quad \times \times \times \quad \times \times \times \quad \times \times \times \quad \times \times \times \quad \times \times \times \quad \times \times \times \quad \times \times \times$

Dec $80 \times \times \times \times \times \times \times \times \times \quad \times \times \times \times \times \times \times \times \times \times \times \times \times \times \times \times \times \times$

Cladostephus spongiosus

Feb $81 \times \times \times \times \times \times \times \times \times \times \times \times \times \times \times \times \times \times \times \times \times \times \times \times \times \times \times$

May $80 \times \times \times \times \times \times \quad \times \times \times \quad \times \times \times \quad \times \times \times \quad \times \times \times \quad \times \times \times \quad \times \times \times \times \times \times$

Jul $81 \times \times \times \quad \times \times \times \quad \times \times \times \times \times \times \quad \times \times \times \quad \times \times \times \quad \times \times \times \quad \times \times \times \quad \times \times \times$

Aug $81 \times \times \times \times \times \times \quad \times \times \times \quad \times \times \times \quad \times \times \times \quad \times \times \times \quad \times \times \times \quad \times \times \times \times \times \times$

Sep $80 \times \times \times \times \times \times \times \times \times \quad \times \times \times \quad \times \times \times \times \times \times \quad \times \times \times \times \times \times \times \times \times$

Oct $81 \times \times \times \quad \times \times \times \quad \times \times \times \times \times \times \times \times \times \times \times \times \times \times \times \quad \times \times \times \times \times \times$ 
Table 4. Rhodophyceae. Temperature tolerance dominant species near Helgoland in two successive years. $\times \times \times=$ plants alive (with measurable photosynthesis)

Species

Temperature during one week $\left({ }^{\circ} \mathrm{C}\right)$

$\begin{array}{llllllll}0 & 5 & 10 & 15 & 18 & 20 & 23 & 25\end{array}$

$28 \quad 30$

Phycodrys rubens

Apr $81 \times \times \times \times \times \times \times \times \times \times \times \times \times \times \times$

May $80 \times \times \times \times \times \times \times \times \times \times \times \times \times \times \times$

Jul $81 \times \times \times \times \times \times \times \times \times \times \times \times \times \times \times \times \times$

Aug $80 \times \times \times \times \times \times \times \times \times \times \times \times \times \times \times \times \times \times$

Aug $81 \times \times \times \times \times \times \times \times \times \times \times \times \times \times \times \times \times \times$

Sep $80 \times \times \times \times \times \times \times \times \times \times \times \times \times \times \times \times \times \times$

Membranoptera alata

Mar $81 \times \times \times \times \times \times \times \times \times \times \times \times \times \times \times \times \times \times$

Jun $80 \times \times \times \times \times \times \times \times \times \times \times \times \times \times \times \times \times$

Jul $81 \times \times \times \times \times \times \times \times \times \times \times \times \times \times \times \times \times \times$

Nov $80 \times \times \times \times \times \times \times \times \times \times \times \times \times \times \times \times \times \times$

Plocamium cartilagineum

Mar $81 \times \times \times \times \times \times \times \times \times \times \times \times \times \times \times \times \times \times$

Aug $81 \times \times \times \times \times \times \times \times \times \times \times \times \times \times \times \times \times \times \times \times$

Rhodomela confervoides

Jan $81 \times \times \times \times \times \times \times \times \times \times \times \times \times \times \times \times \times \times$

Apr $80 \times \times \times \times \times \times \times \times \times \times \times \times \times \times \times \times \times \times$

Jul $80 \times \times \times \times \times \times \times \times \times \times \times \times \times \times \times \times \times \times \times \times \times$

Rhodomela virgata

Jun $81 \times \times \times \times \times \times \times \times \times \times \times \times \times \times \times \times \times \times \times \times$

Aug $80 \times x \times \times \times \times \times \times \times \times \times \times \times \times \times \times \times \times \times \times \times$

Nov $80 \times \times \times \times \times \times \times \times \times \times \times \times \times \times \times \times \times \times \times \times \times$

Delesseria sanguinea

Mar $81 \times \times \times \times \times \times \times \times \times \times \times \times \times \times \times \times \times \times \times \times \times$

Apr $80 \times \times \times \times \times \times \times \times \times \times \times \times \times \times \times \times \times \times \times \times \times$

Apr $81 \times \times \times \times \times \times \times \times \times \times \times \times \times \times \times \times \times \times \times \times \times$

May $80 \times \times \times \quad \times \times \times \quad \times \times \times \quad \times \times \times \quad \times \times \times \quad \times \times \times \times \times \times$

Jun $81 \times \times \times \times \times \times \times \times \times \times \times \times \times \times \times \times \times \times \times \times$

Plumaria elegans

Mar $81 \times \times \times \times \times \times \times \times \times \times \times \times \times \times \times \times \times \times \times \times \times$

Apr $80 \times \times \times \times \times \times \times \times \times \times \times \times \times \times \times \times \times \times \times \times \times$

Jul $81 \times \times \times \times \times \times \times \times \times \times \times \times \times \times \times \times \times \times \times \times \times$

Oct $81 \times \times \times \times \times \times \times \times \times \times \times \times \times \times \times \times \times \times \times \times \times$

Nov $81 \times \times \times \times \times \times \times \times \times \times \times \times \times \times \times \times \times \times \times \times \times$

Cystoclonium purpureum

Apr $81 \times \times \times \times \times \times \times \times \times \times \times \times \times \times \times \times \times \times \times \times \times$

May $80 \times \times \times \times \times \times \times \times \times \times \times \times \times \times \times \times \times \times \times \times \times$

Jul $81 \times \times \times \times \times \times \times \times \times \times \times \times \times \times \times \times \times \times \times \times \times$

Porphyra linearis

Jan $81 \times \times \times \times \times \times \times \times \times \times \times \times \times \times \times \times \times \times \times \times \times$

Porpyhyra umbilicalis

Jan $81 \times \times \times \times \times \times \times \times \times \times \times \times \times \times \times \times \times \times \times \times \times$

May $81 \times \times \times \times \times \times \times \times \times \quad \times \times \times \quad \times \times \times \quad \times \times \times \quad \times \times \times \quad \times \times \times$

Nov $81 \times \times \times \times \times \times \times \times \times \times \times \times \quad \times \times \times \times \times \times \times \times \times \quad \times \times \times$

Bangia fuscopuropurea

Nov $81 \times \times \times \times \times \times \times \times \times \times \times \times \times \times \times \times \times \times \times \times \times \times \times \times$

Dumontia contorta

Jun $80 \times \times \times \times \times \times \times \times \times \times \times \times \times \times \times \times \times \times \times \times \times \times \times \times$

Jul $81 \times \times \times \times \times \times \quad \times \times \times \quad \times \times \times \quad \times \times \times \quad \times \times \times \quad \times \times \times \quad \times \times \times$ 
Table 4. Rhodophyceae (continued)

Species

Temperature during one week $\left({ }^{\circ} \mathrm{C}\right)$

$\begin{array}{llllllllll}0 & 5 & 10 & 15 & 18 & 20 & 23 & 25 & 28 & 30\end{array}$

Rhodochorton floridulum

Oct $81 \times \times \times \times \times \times \times \times \times \times \times \times \times \times \times \times \times \times \times \times \times \times \times \times$

\section{Ceramium rubrum}

Jan $81 \times \times \times \times \times \times \times \times \times \times \times \times \times \times \times \times \times \times \times \times \times$

Jun $80 \times \times \times \times \times \times \times \times \times \times \times \times \times \times \times \times \times \times \times \times \times \times \times$

Jul $81 \times \times \times \times \times \times \times \times \times \times \times \times \times \times \times \times \times \times \times \times \times \times \times \times$

Nov $81 \times \times \times \times \times \times \times \times \times \times \times \times \times \times \times \times \times \times \times \times \times \times \times \times$

Ceramium deslongchampsii

$\operatorname{Jan} 81 \times \times \times \times \times \times \times \times \times \times \times \times \times \times \times \times \times \times \times \times \times \times \times \times \times$

Jul $81 \times \times \times \times \times \times \times \times \times \times \times \times \times \times \times \times \times \times \times \times \times \times$

Polysiphonia urceolata

Mar $81 \times \times \times \times \times \times \times \times \times \times \times \times \times \times \times \times \times \times \times \times \times \times \times \times$

Apr $81 \times \times \times \times \times \times \times \times \times \times \times \times \times \times \times \times \times \times \times \times \times \times \times \times$

Jun $80 \times \times \times \times \times \times \times \times \times \times \times \times \times \times \times \times \times \times \times \times \times \times \times \times \times \times$

Corallina officinalis

May $81 \times \times \times \times \times \times \times \times \times \times \times \times \times \times \times \times \times \times \times \times \times \times \times \times$

Jun $80 \times \times \times \times \times \times \times \times \times \times \times \times \times \times \times \times \times \times \times \times \times \times \times \times \times \times$

Jul $80 \times \times \times \times \times \times \times \times \times \times \times \times \times \times \times \times \times \times \times \times \times \times \times \times \times \times \times$

Jul $81 \times \times \times \times \times \times \times \times \times \times \times \times \times \times \times \quad \times \times \times \quad \times \times \times \quad \times \times \times \times \times \times$

Aug $81 \times \times \times \times \times \times \times \times \times \times \times \times \times \times \times \times \times \times \times \times \times \times \times \times \times \times$

Bonnemaisonia hamifera (Trailliella-phase)

Aug $81 \times \times \times \times \times \times \times \times \times \times \times \times \times \times \times \times \times \times \times \times \times \times \times \times \times \times$

Chondrus crispus

Jan $81 \times \times \times \times \times \times \times \times \times \times \times \times \times \times \times \times \times \times \times \times \times \times \times \times \times \times \times$

Feb $81 \times \times \times \times \times \times \times \times \times \times \times \times \times \times \times \quad \times \times \times \times \times \times \quad \times \times \times \times \times \times$

Apr $80 \times \times \times \times \times \times \times \times \times \times \times \times \times \times \times \times \times \times \times \times \times \times \times \times$

May $81 \times \times \times \times \times \times \times \times \times \times \times \times \times \times \times \times \times \times \times \times \times \times \times \times$

Aug $80 \times \times \times \times \times \times \times \times \times \times \times \times \times \times \times \times \times \times \times \times \times \times \times \times \times \times \times \times \times \times$

Aug $81 \times \times \times \times \times \times \times \times \times \times \times \times \times \times \times \times \times \times \times \times \times \times \times \times \times \times \times \times$

Nov $81 \times \times \times \times \times \times \times \times \times \times \times \times \times \times \times \times \times \times \times \times \times \times \times \times \times \times \times$

Dec $80 \times \times \times \times \times \times \times \times \times \times \times \times \times \times \times \times \times \times \times \times \times \times \times \times \times \times \times$

Ahnfeltia plicata

Jul $80 \times \times \times \times \times \times \times \times \times \times \times \times \times \times \times \times \times \times \times \times \times \times \times \times \times \times$ Jul $81 \times \times \times \times \times \times \times \times \times \times \times \times \times \times \times \times \times \times \times \times \times \times \times \times \times \times \times$

Aug $81 \quad x \times \times \times \times \times \times \times \times \quad \times \times \times \quad \times \times \times \quad \times \times \times \quad \times \times \times \quad \times \times \times \quad \times \times \times$

\section{Phyllophora truncata}

May $80 \times \times \times \times \times \times \times \times \times \times \times \times \quad \times \times \times \times \times \times \times \times \times \times \times \times \times \times \times$ Jun $80 \times \times \times \times \times \times \times \times \times \times \times \times \times \times \times \times \times \times \times \times \times \times \times \times \times \times \times$ Aug $81 \times \times \times \times \times \times \times \times \times \times \times \times \times \times \times \quad \times \times \times \quad \times \times \times \times \times \times \times \times \times$

Phyllophora pseudoceranoides

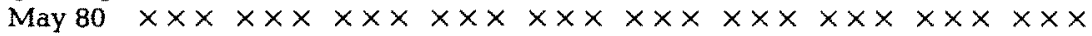
Jul $80 \times \times \times \times \times \times \times \times \times \times \times \times \times \times \times \times \times \times \times \times \times \times \times \times \times \times \times \times \times \times$ Jul $81 \times \times \times \times \times \times \times \times \times \times \times \times \times \times \times \times \times \times \times \times \times \times \times \times \times \times \times \times$

\section{Polyides rotundus}

May $80 \times \times \times \times \times \times \times \times \times \times \times \times \times \times \times \times \times \times \times \times \times \times \times \times \times \times \times$

Jul $80 \times \times \times \times \times \times \times \times \times \times \times \times \times \times \times \times \times \times \times \times \times \times \times \times \times \times \times \times \times \times$

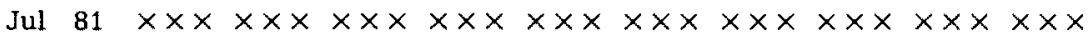

Sep $80 \times \times \times \times \times \times \times \times \times \times \times \times \times \times \times \times \times \times \times \times \times \times \times \times \times \times \times \times \times \times$ 
Table 5. Chlorophyceae. Temperature tolerance of dominant species near Helgoland in two successive years. $\times \times \times=$ plants alive (with measurable photosynthesis)

Species

Temperature during one week $\left({ }^{\circ} \mathrm{C}\right)$

\begin{tabular}{lllllllllll}
0 & 5 & 10 & 15 & 18 & 20 & 23 & 25 & 28 & 30 & 33 \\
\hline
\end{tabular}

Monostroma undulatum

Apr $80 \times \times \times \times \times \times \times \times \times$

May $81 \times \times \times \times \times \times \times \times \times$

Acrosiphonia arcta

Feb $81 \times \times \times \times \times \times \times \times \times \times \times \times \times \times \times \times \times$

Apr $81 \times \times \times \times \times \times \times \times \times \times \times \times \times \times \times \times \times \times$

Acrosiphonia sonderi

Apr $80 \times \times \times \times \times \times \times \times \times \times \times \times \times \times \times \times \times$

Blidingia minima

May $81 \times \times \times \times \times \times \times \times \times \times \times \times \times \times \times \times \times \times \times \times \times$

Jun $80 \times \times \times \times \times \times \times \times \times \times \times \times \times \times \times \times \times \times \times \times \times$

Aug $81 \times \times \times \times \times \times \times \times \times \times \times \times \times \times \times \times \times \times \times \times \times \times \times \times$

Oct $81 \times \times \times \times \times \times \times \times \times \times \times \times \times \times \times \times \times \times \times \times \times \times \times \times$

Dec $81 \times \times \times \times \times \times \times \times \times \times \times \times \times \times \times \times \times \times \times \times \times \times \times \times$

Chaetomorpha melagonium

Jul $80 \times \times \times \times \times \times \times \times \times \times \times \times \times \times \times \times \times \times \times \times \times \times \times \times$

Oct $81 \times \times \times \times \times \times \times \times \times \times \times \times \quad \times \times \times \quad \times \times \times \times \times \times \times \times \times$

Nov $81 \times \times \times \times \times \times \times \times \times \times \times \times \times \times \times \quad \times \times \times \times \times \times \quad \times \times \times$

Chaetomorpha tortuosa

Jun $80 \times \times \times \times \times \times \times \times \times \times \times \times \times \times \times \times \times \times \times \times \times \times \times \times$

Aug $81 \times \times \times \times \times \times \times \times \times \times \times \times \times \times \times \times \times \times \times \times \times \times \times \times$

Oct $81 \times \times \times \times \times \times \times \times \times \times \times \times \times \times \times \times \times \times \times \times \times \times \times \times$

Rhizoclonium ripàrium

Aug $81 \times \times \times \times \times \times \times \times \times \times \times \times \times \times \times \times \times \times \times \times \times \times \times \times \times \times \times$

Cladophora rupestris

May $81 \times \times \times \times \times \times \times \times \times \times \times \times \times \times \times \times \times \times \times \times \times \times \times \times \times \times \times$

Jul $81 \times \times \times \times \times \times \times \times \times \times \times \times \times \times \times \times \times \times \times \times \times \times \times \times \times \times \times$

Aug $81 \times \times \times \times \times \times \times \times \times \times \times \times \times \times \times \times \times \times \times \times \times \times \times \times \times \times \times$

Sep $80 \times \times \times \times \times \times \times \times \times \times \times \times \times \times \times \times \times \times \times \times \times \times \times \times \times \times \times$

Oct $81 \times \times \times \times \times \times \times \times \times \times \times \times \times \times \times \times \times \times \times \times \times \times \times \times \times \times \times$

Nov $81 \times \times \times \times \times \times \times \times \times \times \times \times \times \times \times \times \times \times \times \times \times \times \times \times \times \times \times$

Ulva lactuca

Apr $80 \times \times \times \times \times \times \times \times \times \times \times \times \times \times \times \times \times \times \times \times \times \times \times \times \times \times \times$

May $80 \times \times \times \times \times \times \times \times \times \times \times \times \times \times \times \times \times \times \times \times \times \times \times \times \times \times \times$

May $81 \times \times \times \times \times \times \times \times \times \times \times \times \times \times \times \times \times \times \times \times \times \times \times \times \times \times \times$

Jul $81 \times \times \times \times \times \times \times \times \times \times \times \times \times \times \times \times \times \times \times \times \times \times \times \times \times \times \times$

Aug $80 \times \times \times \times \times \times \times \times \times \times \times \times \times \times \times \times \times \times \times \times \times \times \times \times \times \times \times$

Aug $81 \times \times \times \times \times \times \times \times \times \times \times \times \times \times \times \quad \times \times \times \times \times \times \times \times \times \times \times \times$

Nov $81 \times \times \times \times \times \times \times \times \times \times \times \times \times \times \times \times \times \times \times \times \times \times \times \times \times \times \times$

Ulva curvata

Nov $81 \times \times \times \times \times \times \times \times \times \times \times \times \times \times \times \times \times \times \times \times \times \times \times \times \times$

Enteromorpha prolifera

May $81 \times \times \times \times \times \times \times \times \times \times \times \times \times \times \times \times \times \times \times \times \times \times \times \times \times \times \times$

Aug $81 \times \times \times \times \times \times \times \times \times \times \times \times \times \times \times \times \times \times \times \times \times \times \times \times \times \times \times \times \times \times$

Codium fragile

Jan $81 \times \times \times \times \times \times \times \times \times \times \times \times \times \times \times \times \times \times \times \times \times \times \times \times \times \times \times$

Apr $80 \times \times \times \times \times \times \times \times \times \times \times \times \times \times \times \times \times \times \times \times \times \times \times \times \times \times \times$

Aug $81 \times \times \times \times \times \times \times \times \times \times \times \times \times \times \times \times \times \times \times \times \times \times \times \times \times \times \times \times \times \times$

Oct $81 \times \times \times \times \times \times \times \times \times \times \times \times \times \times \times \times \times \times \times \times \times \times \times \times \times \times \times \times \times \times$

Bryopsis hypnoides

Jun 81 $\times \times \times \times \times \times \times \times \times \times \times \times \times \times \times \times \times \times \times \times \times \times \times \times \times \times \times \times \times \times$ Jul $81 \times \times \times \times \times \times \times \times \times \times \times \times \quad \times \times \times \quad \times \times \times \quad \times \times \times \quad \times \times \times \times \times \times \times \times \times$ 


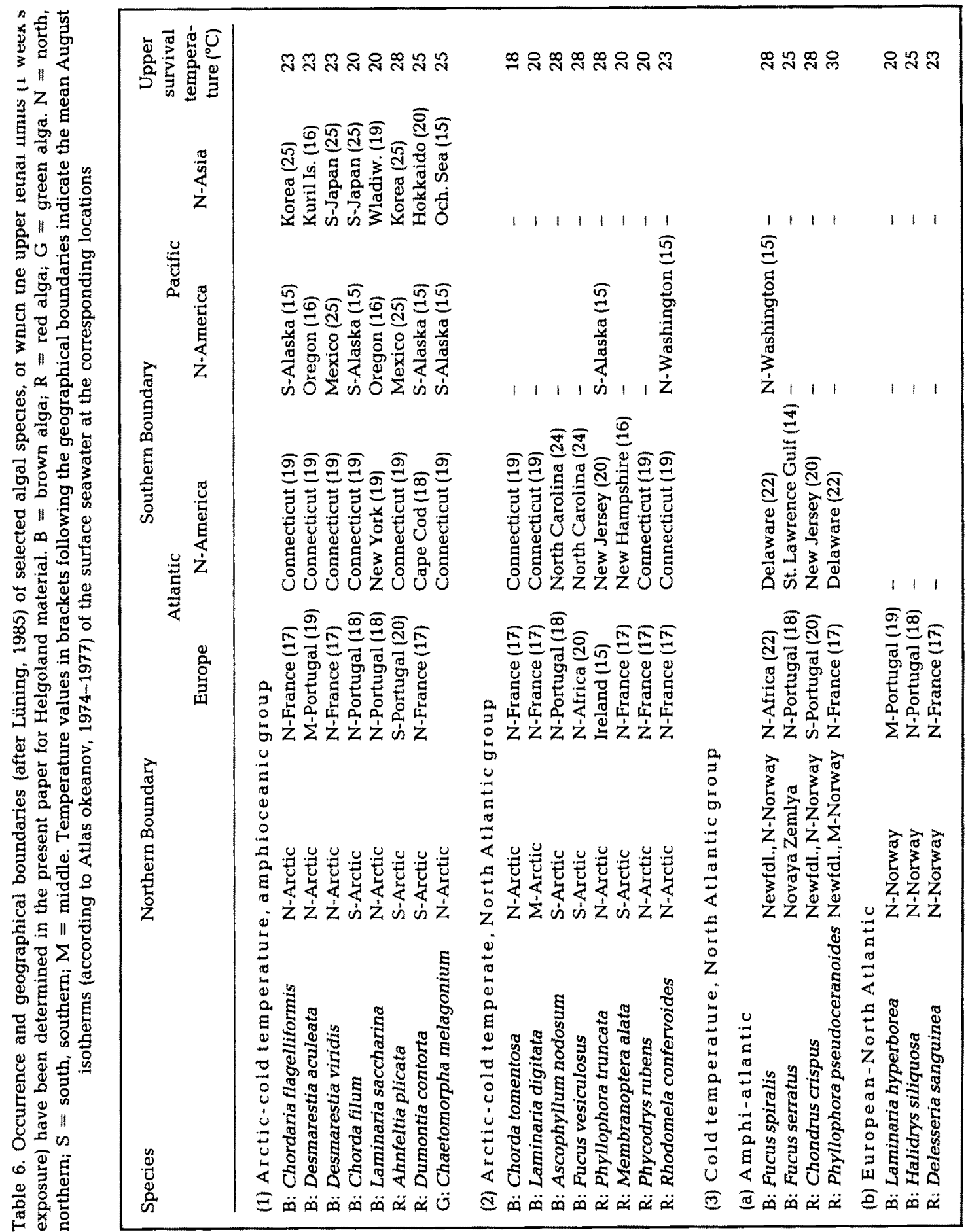


Montfort et al. (1957) and are in accord with the well-known higher heat sensitivity of actively growing plant tissue in general (Larcher, 1980). The occurrence of a summer peak in heat tolerance is also widespread among higher plants ("S-type" response; see Larcher et al., 1973; Kappen, 1981). As may be seen from the data in Tables 3 and 2, the seasonal course of temperature tolerance of $D$. aculeata follows the seasonal course of seawater temperature. It remains at its summer maximum at least until November, when the seawater temperature has dropped already to $9^{\circ} \mathrm{C}$.

Seasonal shifts of heat tolerance by $2^{\circ} \mathrm{C}$ were also observed in a few red and green algal species (Tables 4-5), and probably more examples might have been detected, if experiments had been conducted more frequently during the year. In general, however, seasonal differences in heat tolerance of $5^{\circ} \mathrm{C}$ are obviously not surpassed, and this is exactly the magnitude which has been found for seasonal shifts of heat tolerance in many other plants, which contrast to the much bigger seasonal changes in cold tolerance (Larcher et al., 1973). Fucus vesiculosus, which did not show a seasonal shift of heat tolerance in the present investigation, withstood in summer $-30^{\circ} \mathrm{C}$, but $-45^{\circ} \mathrm{C}$ in winter, as determined for material from Long Island by Parker (1960).

In view of the relatively small seasonal changes of heat tolerance it is clear that the species-specific differences in heat tolerance (Tables 3-5) are genetically fixed, not only for species, but also for higher taxonomic entities. Beginning with the Phaeophyceae (Table 3), the upper survival limits range from $18^{\circ} \mathrm{C}$ in case of the spring annual Chorda tomentosa or from $20^{\circ} \mathrm{C}$ in case of the other representatives of the Laminariales to $28^{\circ} \mathrm{C}$ in the representatives of the Fucales, except for Fucus serratus and Halidrys siliquosa, which survive only $25^{\circ} \mathrm{C}$. Here the striking difference in regard to the upper survival temperatures between the representatives of the two brown algal orders, emphasized already by Setchell (1920), becomes evident. The investigated Helgoland representatives of the Desmarestiales, the Scytosiphonales and the Sphacelariales are characterized by upper survival temperatures at $23-25^{\circ} \mathrm{C}$.

Among the Rhodophyceae (Table 4) the most sensitive species are the representatives of the Delesseriaceae which only survive $20^{\circ} \mathrm{C}$ (Phycodrys rubens, Membranoptera alata) or $23^{\circ} \mathrm{C}$ (Delesseria sanguinea), the latter temperature also marking the upper survival limit of the Helgoland Rhodomela spp., whereas the Ceramium spp. survive $25^{\circ} \mathrm{C}$. In contrast, the representatives of the Gigartinales (Phyllophora spp., Ahnfeltia plicata, Chondrus crispus, Polyides rotundus) are all found at the upper end of the temperature survival scale, with survival temperatures of $28-30^{\circ} \mathrm{C}$. Biebl (1958), when investigating the temperature tolerance of algae from the coast of Brittany, had found an upper survival temperature of $27^{\circ} \mathrm{C}$ for Phycodrys rubens, Membranoptera alata, and Delesseria sanguinea, thus $7^{\circ} \mathrm{C}$ or $4^{\circ} \mathrm{C}(D$. sanguinea) higher than in the present investigation. This difference is probably artificial and due to the short exposure time of 12 h used by Biebl.

The most sensitive species of the Chlorophyceae in the Helgoland seaweed flora (Table 5) is the early spring annual Monostroma undulatum which survives $10^{\circ} \mathrm{C}$, but dies at $15^{\circ} \mathrm{C}$. Next on the scale are the Acrosiphonia spp., again spring annuals, with upper survival limits of $20^{\circ} \mathrm{C}$, the Chaetomorpha spp., which survive $25^{\circ} \mathrm{C}$, and finally a somewhat bigger, heat-tolerant group with upper survival limits at $28^{\circ} \mathrm{C}$ (Cladophora rupestris, Ulva spp.) or finally at $30^{\circ} \mathrm{C}$ (Enteromorpha prolifera, Codium fragile, and Bryopsis hypnoides). 
The morphologically larger of the 54 investigated species, i.e. 23 species, are again listed in Table 6 with regard to their affiliation to four geographical distribution groups, according to their occurrence in the Arctic, in the North Atlantic, and in the North Pacific. The fact that the 16 species listed as occurring also in the Arctic (groups 1 and 2) display similar upper survival limits as the species with cold temperature distributions (groups $3 a$ and $3 b$ ) indicates that the species which invaded the Arctic after the last glaciation can hardly be characterized by reduced upper survival temperatures, although data on temperature tolerance of seaweeds in the Arctic is still largely lacking. The few data on temperature tolerance of Arctic seaweeds supports the view that time has been too short in the Arctic for the formation of real cold water algae which lose their ability to survive at temperatures above 18 or $20^{\circ} \mathrm{C}$. Biebl (1968; Table 1) found as a result of short $(12 \mathrm{~h})$ exposures to varying temperatures that Agarum cribrosum and Laminaria saccharina from West Greenland had upper survival temperatures of $24^{\circ} \mathrm{C}$. Bolton \& Lüning (1982) detected an upper survival limit of $23^{\circ} \mathrm{C}$ for Laminaria saccharina subsp. longicruris gametophytes from the Canadian Arctic, or from Newfoundland, exposed for 4 weeks to varying temperatures. Even the gametophytes of the arcticendemic $L$. solidungula survive $18^{\circ} \mathrm{C}$, however not $20^{\circ} \mathrm{C}$, according to the latter authors. A more pronounced adaption to cold conditions is to be expected in the subantarctic and antarctic regions with a longer history as cold water habitats than the Arctic, and the only result in this respect, the death of Durvillaea antarctica at $14{ }^{\circ} \mathrm{C}$ (Delépine \& Asensi, 1976) points in this direction. Also the fact that tropical sublittoral algae may have a lower lethal temperature as high as $14^{\circ} \mathrm{C}$, as found by Biebl (1962; Table 1), shows that seaweeds do change their temperature characteristics dramatically given a long enough geological time period.

Of the species listed in Table 6, several cases have been analysed in detail by Hoek (1982a, 1982b) with regard to their geographical distribution along seawater isotherms as compared to the temperature tolerances, and temperature demands for growth or reproduction, of a particular species. These species are Chondrus crispus, Desmarestia aculeata, D. viridis (van den Hoek, 1982a), Laminaria saccharina, L. digitata, and L. hyperborea (van den Hoek, 1982b). Inasmuch as amphi-atlantic species are concerned, usually composite boundaries seem to limit the distribution to the south, i.e. upper survival limits determine the southern boundary of occurrence on the American side, and temperature requirements for growth and/or reproduction on the European side of the Atlantic. Similar analyses may be also possible in the future for the rest of the species listed in Tables $2-5$ which only provide information on temperature tolerance. However, to achieve this goal, more data about the species' temperature tolerance from other coasts is required, as well as about the temperature requirements for growth and reproduction.

The possibility that Chordaria flagelliformis, Chorda filum, and $D$. viridis may differ with regard to their upper survival temperatures in the North Pacific and in the North Atlantic is indicated by the finding that the upper survival temperature of $23^{\circ} \mathrm{C}$, as determined for these species in Helgoland material, would not enable the Pacific counterparts to reach their actual southern limits (Table 6). 


\section{LITERATURE CITED}

Atlas okeanov, 1974-1977. Ministerstvo Oborony SSSR, Voenno-Morskoj Flot, 1-2.

Biebl, R, 1958. Temperatur- und osmotische Resistenz von Meeresalgen der bretonischen Küste. Protoplasma 50, 217-242.

Biebl, R., 1962. Temperaturresistenz tropischer Meeresalgen. (Verglichen mit jener von Algen in temperierten Meeresgebieten.) - Botanica mar. 4, 241-254.

Biebl, R., 1968. Uber Wärmehaushalt und Temperaturresistenz arktischer Pflanzen in Westgrönland. - Flora (Abt. B) 157, 327-354.

Biebl, R., 1970. Vergleichende Untersuchungen zur Temperaturresistenz von Meeresalgen entlang der pazifischen Küste Nordamerikas. - Protoplasma 69, 61-83.

Bolton, J. J., 1983. Ecoclinal variation in Ectocarpus siliculosus (Phaeophyceae) with respect to temperature growth optima and survival limits. - Mar. Biol. 73, 131-138.

Bolton, J. J. \& Lüning, K., 1982. Optimal growth and maximal survival temperatures of Atlantic Laminaria species (Phaeophyta) in culture. - Mar. Biol. 66, 89-94.

Briggs, J. C., 1974. Marine zoogeography. McGraw-Hill, New York, 475 pp.

Cambridge, M., Breeman, A. M., van Oosterwijk, R. \& Hoek, C. van den, 1984. Temperature responses of some North Atlantic Cladophora species (Chlorophyceae) in relation to their geographical distribution. - Helgoländer Meeresunters. 38, 349-363.

Delépine, R. \& Asensi, A., 1976. Quelques données expérimentales sur l'écophysiologie de DurvilIaea antarctica (Cham.) Hariot (Péophycées). - Bull. Soc. phycol. France 21, 65-80.

Frakes, L. A., 1979. Climates throughout geologic time. Elsevier, Amsterdam, 310 pp.

Hoek, C. van den, 1975. Phytogeographic provinces along the coasts of the northern Atlantic Ocean. - Phycologia 14, 317-330.

Hoek, C. van den, 1982a. Phytogeographic distribution groups of benthic marine algae in the North Atlantic Ocean. - Helgoländer Meeresunters. 35, 153-214.

Hoek, C. van den, $1982 \mathrm{~b}$. The distribution of benthic marine algae in relation to the temperature regulation of their life histories. - Biol. J. Linn. Soc. Lond. 18, 81-144.

Kappen, L., 1981. Ecological significance of resistance to high temperature. In: Encyclopedia of plant physiology. (N. S.), Ed. by O. L. Lange, P. S. Nobel, C. B. Osmond \& H. Ziegler. Springer, Berlin, 12A, 439-474.

Lampe, H., 1935. Die Temperatureinstellung des Stoffgewinns bei Meeresalgen als plasmatische Anpassung. - Protoplasma 23, 534-578.

Larcher, W., 1980. Physiological plant ecology. Springer, Berlin, 303 pp.

Larcher, W., Heber, U. \& Santarius, K. A., 1973. Plants: Limiting temperatures for life functions. In: Temperature and life. Ed. by H. Precht, J., Christophersen, H. Hensel, \& W. Larcher. Springer, Berlin, 195-231.

Lüning, K., 1985. Meeresbotanik. Thieme, Stuttgart (in press).

McLachlan, J. \& Bird, C. J., 1984. Geographical and experimental assessment of the distribution of species of Gracilaria Grev. (Rhodophyta: Gigartinales) in relation of temperature, - Helgoländer Meeresunters. 37, 319-334.

Montfort, C., Ried, A. \& Ried, I., 1957. Abstufungen der funktionellen Wärmeresistenz bei Meeresalgen in ihren Beziehungen zu Umwelt und Erbgut. - Biol. Zbl. 76, 257-289.

Parker, J., 1960. Seasonal changes in cold-hardiness of Fucus vesiculosus. - Biol. Bull. mar. biol. Lab. Woods Hole, 119, 474-478.

Rietema, H. \& Hoek, C. van den, 1984. Search for possible latitudinal ecotypes in Dumontia contorta (S. G. Gmel.) Rupr. (Rhodophyta). - Helgoländer Meeresunters. 38, 389-399.

Schramm, W., 1968. Okologisch-physiologische Untersuchungen zur Austrocknungs- und Temperaturresistenz an Fucus vesiculosus L. der westlichen Ostsee. - Int. Revue ges. Hydrobiol. 53, $469-510$.

Setchell, W. A., 1920. Stenothermy and zone-invasion. - Am. Nat. 54, 385-397.

Yarish, C., Breeman, A. M. \& Hoek, C. van den, 1984. Temperature, light, and photoperiod responses of some northeast American and west European endemic Rhodophytes in relation to their geographical distribution. - Helgoländer Meeresunters. 38, 273-304. 